\title{
Visibility Monitoring using Conventional Roadside Cameras - Emerging Applications
}

\author{
Raouf Babari ${ }^{\mathrm{a}}$, Nicolas Hautière ${ }^{\mathrm{a}}$, Éric Dumont $^{\mathrm{a}}$, Nicolas Paparoditis ${ }^{\mathrm{b}}$, James Misener $^{\mathrm{c}}$ \\ ${ }^{a}$ Université Paris-Est, Ifsttar, IM, LEPSIS, 58 boulevard Lefebvre, F-75015 Paris \\ ${ }^{b}$ Université Paris-Est, IGN, MATIS, 73 avenue de Paris, F-94160 Saint-Mandé \\ ${ }^{c}$ Booz Hallen Hamilton Inc., 101 California Street, Suite 3300, San Francisco, CA
}

\begin{abstract}
The measurement of atmospheric visibility is an important element for road and air transportation safety. We propose in this paper a novel estimator of the atmospheric visibility by already existing conventional highway cameras, with a technique based on the gradient magnitude selected by applying Lambert's law with respect to changes in lighting conditions. The response of this estimator is calibrated by non-linear regression with data from a visibility meter installed in a test site which has been instrumented with a camera. Through our technique, atmospheric visibility estimates are obtained with an average error of $30 \%$ for images taken in the day, with sky luminance between 10 to $8,000 \mathrm{~cd} \cdot \mathrm{m}^{-2}$ and visibility distances up to 15 $\mathrm{km}$. Our results allow us to envision practical implementation on roadsides in the near future to determine local visibility for the benefit of road safety, meteorological observation and air quality monitoring.
\end{abstract}

Keywords: camera, atmospheric visibility, road safety, meteorological observation, air quality, Intelligent Transportation Systems

\section{Introduction}

In the presence of dust, smoke, fog, haze or pollution, meteorological visibility is reduced. This constitutes a common and vexing transportation problem for different public authorities in multiple countries throughout the world.

First, low visibility is obviously a problem of traffic safety. It can be caused by dust, smoke or fog. Dust storms potentially reduce the visibility to zero and cause dramatic pile-ups. These phenomena are likely to occur in dry agricultural areas subject to land erosion, like the I-5 in California's San Joaquin Valley in 1991 (164 vehicles involved, 151 injured, 17 dead) (Pauley et al., 1996) or the A19 Mecklenburg-Vorpommern in Germany in 2011 (82 vehicles involved, 100 injured, 8 dead) (Hunfeld et al., 2011). Smoke caused by factories or fires in the vicinity of highways is also a dramatic source of reduction of the visibility (AbdelAty et al., 2011). Road crashes which occur in fog are generally more severe than the average crash (AbdelAty et al., 2011). According to NOAA (Whiffen et al., 2004), in the United States there are approximately 700 annual fog-related fatalities, defined as occurring when visibility is less than $\frac{1}{4}$ mile (400 meters). Fog constitutes an equally important issue in France, a smaller country, with over 100 annual fatalities attributed to low visibility. Indeed, fog causes similar and significant problems on Northern America and French highways. The combination of fog and smoke is even worse. It was the cause of dramatic pile-ups in France, e.g. on the A10 in 2002 near Coulombiers (58 vehicles involved, 40 injured, 8 dead). Even if both phenomena differ in their origin, their mutual effect on visibility is exponential, which leads to areas with zero visibility. It should be stressed that the solution lies not necessarily in better low visibility detection but in driver response to fog that is detected. 
Indeed, the behavior of drivers in fog is often inappropriate (e.g. reduced headways, altered reaction times) but to understand the origins of these dangerous behaviors is difficult (Kang et al., 2008). Different countermeasures have been tested to mitigate the impact of critically reduced visibility (Shepard, 1996). The California San Joaquin and Sacramento Valley regions are particularly adequate test-beds for such measures, because of the well-known Tule fog phenomenon. In the Stockton area of Caltrans District 10, the Caltrans Automated Warning System (CAWS) employs roadside weather stations and visibility meters to provide automated detection (Mac Carley, 2005). In District 6, Caltrans has installed the "Fog Pilot" system, which provides a high-technology solution every $\frac{1}{4}$ mile along a 12-mile (20-km) portion of State Route 99.

In addition to the safety problem, reduced visibility is cause of delays and disruption in air, sea and ground transportation for passengers and freight. On freeways, massive pile-ups create exceptional traffic congestions which sometimes force the operator to momentarily close the road. Fog-related road closures are not an uncommon subject for news headlines. For example, the Heathrow airport was blocked for three days during the 2006 Christmas period. Such events have of course important economic impacts (Pejovic et al., 2009). According to Perry and Symons (1991), in 1974 fog was estimated to have cost over roughly $£ 120$ millions at 2010 prices on the roads of Great Britain. This figure includes the cost of medical treatment, damage to vehicles and property, as well as the administrative costs of police, services and insurance, but they do not include the cost of delays to vehicles not directly involved in the accident.

Moreover, reduced visibility also has environmental significance. Visibility is generally valued for environmental and aesthetic reasons that are difficult to express or quantify. Except for American national parks (Committee on Haze in National Parks and Wilderness Areas, 1993) and regulations on freeway advertisements, there are few places where visibility is considered a protected resource. Impaired visibility is also a symptom of environmental problems because it is evidence of air pollution (Hyslop, 2009). In addition, it has been shown that impaired visibility and mortality are related (Thach et al., 2010). According to the authors, visibility provides a useful proxy for the assessment of environmental health risks from ambient air pollutants and a valid approach for the assessment of the public health impacts of air pollution where pollutant monitoring data are scarce.

An ability to accurately monitor visibility helps resolve these problems. Important transportation facilities where safety is critical, such as airports, are generally instrumented for monitoring visibility with devices that are expensive and hence, scarce. Cost is precisely the reason why highway meteorological stations are seldom equipped with visibility metering devices. In this context, using already existing and ubiquitous highway cameras is of great interest, as these are low cost sensors already deployed for other purposes such as traffic monitoring (Jacobs et al., 2009). Furthermore, introducing new functionalities into roadside cameras will make them multipurpose and thus more cost-effective, easing their deployment along the roads.

In the United States, this potential has been identified by US DOT and was evaluated in the CLARUS Initiative (Hallowell et al., 2007), and these efforts may continue with the US DOT IntelliDrive program. In France, a similar initiative has been launched between Ifsttar (French institute of science and technology for transport, development and networks), Météo France (French National Weather Service) and IGN (French National Geographical Institute), three public research institutes dealing respectively with road operation, weather monitoring and forecasting, and geography and cartography. The French initiative aims at assessing the potential of highway cameras to monitor visibility for different applications ranging from safety hazard detection to air quality monitoring. This topic has also been a matter of discussion and of potential collaboration between Ifsttar and California Partners for Advanced Transit and Highways (PATH) at Berkeley University. In the future, such initiatives might make it possible to monitor visibility reduction at the scale 
of a road itinerary. Prediction, which will soon be possible for airports (Roquelaure et al., 2009), might be envisioned.

\section{Background}

\subsection{Visibility Measurement}

Reduced visibility in the atmosphere is directly related to light scattering by air molecules and airborne particles. This tenet of physics is the basis of the operating principle of visibility meters. There are two types of instruments for measuring atmospheric visibility: transmissometers and scatterometers. The transmitometer extrapolates the attenuation of a light beam emitted from a source to a receiver at a known path length in order to estimate the distance for which the emitted light is attenuated by $95 \%$. The transmitometer is also used to calibrate the scatterometer. A scatterometer assesses the dispersion of a light beam. Visibility meters can measure the meteorological visibility distance up to a few tens of kilometers with an accuracy of $10 \%$. Some studies seek to exploit the photosensitive cells of fixed cameras to measure the meteorological visibility.

\subsection{The Potential of the CCTV Camera Networks: The French Case}

The annual statistics on fog occurrence in France, i.e. episodes where the meteorological visibility distance is lower than 1000 meters, are shown in Figure 1(a). Such a data collection is obtained thanks to 60 weather stations distributed over the entire territory (see blue points in the Figure 1(a)). As such, these observations cannot be used for predicting and warning road authorities. Indeed, the local nature of this phenomenon is not compatible with the current capacity of meteorological agencies to monitor it accurately.

A survey has been conducted on the French motorway networks to estimate the potential of existing CCTV networks to monitor the visibility: In 2009, the French motorway network was 8,372 km long and was equipped with approximately 2,000 cameras. Accounting for the fact that some cameras are grouped together and some are dedicated to tunnel safety, a potential of 1,000 cameras available to monitor the weather is estimated. The French highway network is also equipped with cameras but they are less numerous. This whole network covers the territory quite uniformly, see Figure 1(b). Consequently, a sensor network along the roadside constitutes a relevant mesh able to feed meteorological centers with geolocalized data. Another important information concerns the type of installed camera. Most cameras have a low resolution, from 470TVL to 570TVL. This lead us to choose a similar camera type in our study. Furthermore, introducing new functionalities into roadside cameras will make them multipurpose and thus more cost-effective, easing the deployment of these cameras along the roads.

\subsection{Related Research}

There are several general approaches to measuring meteorological visibility with a camera. The first is to detect the contrast of the most distant targets in a scene. For road safety, and visibility distances below $400 \mathrm{~m}$, Hautière et al. (2008) assume that the road is flat. They calculate all contrasts above 5\% for objects obtained from the camera images. Using the geometric projection, they then estimate the distance to the farthest visible object with an intrinsic error of $10 \%$. In another study by Bäumer et al. (2008), in a panoramic scene, extract gradients of targets whose distances are known based on a 2-dimensional map. In this work, ranges are longer because in meteorology, visibility distances are of the order of $10 \mathrm{~km}$.

The second general approach to measuring meteorological visibility is based on machine learning, and requires a calibration phase with meteorological data collected with a visibility meter for several days and 


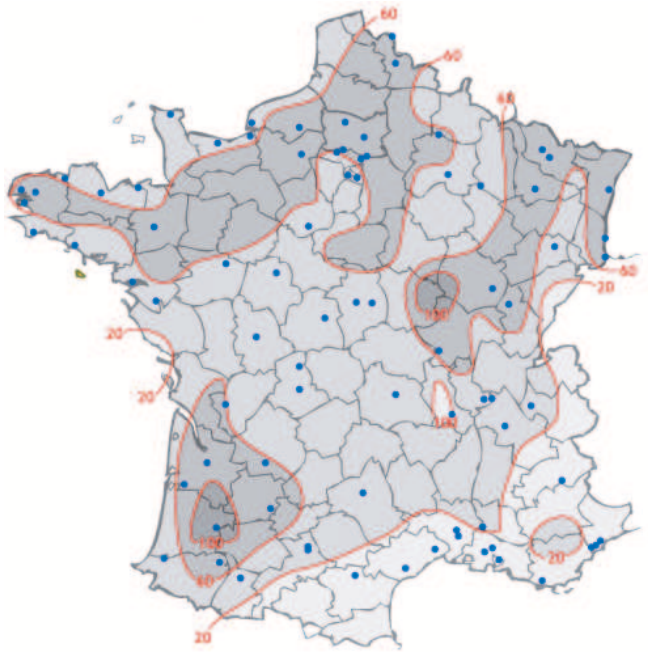

(a)

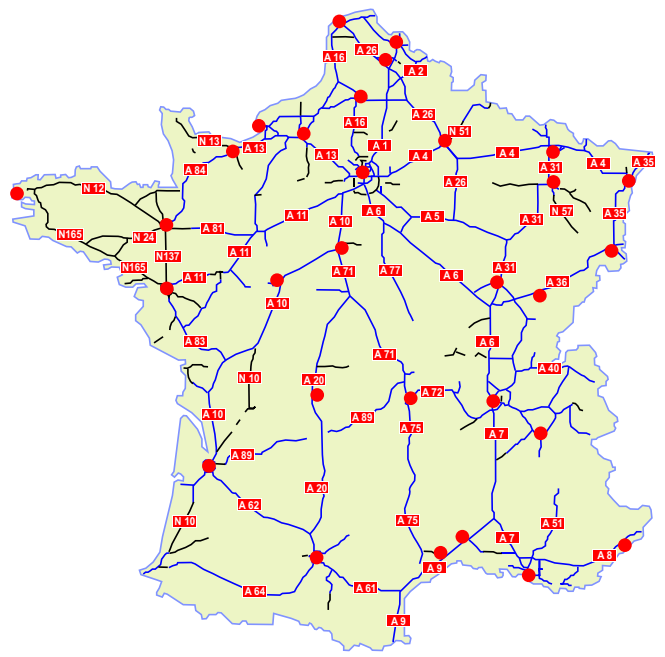

(b)

Figure 1: Existing meteorological visibility observations compared to the potential of CCTV networks: (a) Average number of foggy days observed in France between 1971 and 2000. The location of automated weather stations which collect visibility measurements are depicted by blue points. (b) The French main highway network is well spread on the whole territory and holds a potential of 1,000 CCTV cameras which are already available to monitor the meteorological visibility distance.

in different visibility conditions. In his study, Hallowell et al. (2007) exploit the road surveillance video camera network by proposing a fuzzy logic-based method which identifies four classes of visibility using image information. Other approaches which exploit machine learning seek to find the frequency response characteristics linking the image with visibility data. Indeed, Xie et al. (2008) and Liaw et al. (2010) seek the linear correlation between some indicator of contrast and meteorological visibility data. Xie et al. (2008) applies a low pass filter to the Fourier transform of the image. Hagiwara et al. (2006) also propose the WIPS frequency operator (Weighted Intensity Power Spectrum) which was proven to be well correlated with human perception. Liaw et al. (2010) acquire images at midday, seeking ways to reduce the influence of changing illumination.

The approach in this paper belongs to the second category. Indeed, an image-based estimator using a fixed Closed Circuit Television (CCTV) camera is proposed. Estimation results can be verified with meteorological visibility ground truth data collected with meteorological instruments. Unlike previous approaches, this one is stable to illumination change and therefore more indicative of the visibility. This article is organized as follows: Section 2 establishes the link between visibility and the gradient in the image; Section 3 clarifies the robustness of the approach; the results are presented in Section 4; a discussion follows, from which conclusions are drawn.

\section{Method}

\subsection{Reduction in Visibility by Scattering}

Although, the word "visibility" seems to be trivial, a more precise definition dedicated to meteorology is established through the theory of Koschmieder (Middleton, 1952) which provides an analytic expression of the luminance $L$ of an object observed from a distance $d$ through an atmosphere with an extinction 
coefficient $k$. This is given by Equation 1:

$$
L=L_{0} e^{-k d}+L_{b}\left(1-e^{-k d}\right)
$$

The physical luminance of an object $L$ reaching the camera is a linear combination of the intrinsic luminance of the object $L_{0}$ and the luminance of the sky $L_{b}$. The linear coefficient is an exponential function of the optical depth $k d$ of the atmosphere which lies between the object and the camera (following Beer-Lambert law). From Equation 1, Duntley (Middleton, 1952) derived a contrast attenuation law:

$$
C=\left(\frac{L_{0}-L_{b}}{L_{b}}\right) e^{-k d}=C_{0} e^{-k d}
$$

The quantity $C$ denotes the apparent contrast at a distance $d$ of an object of luminance $L$ against the sky in the background with a luminance $L_{b} . C_{0}$ is the intrinsic contrast of this object. The International Commission on Illumination (CIE) recommends a threshold contrast of 5\% to define visibility, so the meteorological visibility $V_{M e t}$, expressed in Equation 3, is defined as the distance for which a black object $\left(C_{0}=-1\right)$ has a $5 \%$ contrast against the sky (CIE, 1987):

$$
V_{\mathrm{Met}}=-\frac{1}{k} \log (0.05) \approx \frac{3}{k}
$$

\subsection{Stability of Contrast in Lambertian Zones}

In order to work with pixel intensity (or gray level) values given by a camera, the arguable assumption can be made that the response of the sensor is linear with a slope $\alpha$. The intensity $I$ of an object in the image can be expressed according to the value $L$ of its physical luminance as shown in Equation 4:

$$
I=\alpha L
$$

Using Equation 4 with Koschmieder law in Equation 1 yields the following relation between the intensity $I$ of a pixel, the optical depth $k d$ of the atmosphere between the camera and the object in the direction subtended by this pixel, and the intensity $A_{\infty}$ of the background sky:

$$
I=I_{0} e^{-k d}+A_{\infty}\left(1-e^{-k d}\right)
$$

Let us introduce the texture contrast $C_{\text {Texture }}$, defined for two adjacent points of intensity $I_{1}$ and $I_{2}$ found at the same distance $d_{1}=d_{2}=d$. Then from Equation 5, Equation 6 is obtained:

$$
C_{\text {Texture }}=\frac{I_{2}-I_{1}}{A_{\infty}}=\left(\frac{I_{02}-I_{01}}{A_{\infty}}\right) e^{-k d}
$$

The luminance of an object results from the reflection of both direct sunlight and light scattered through the atmosphere onto its surface. For objects with rough (and therefore Lambertian) surfaces, the reflected part of illuminance $E$ is scattered evenly in all directions, and the luminance $L$ is directly related to the albedo $\rho$ of the surface material. In that case $\rho$ is the diffuse reflection factor of the object. This is expressed by Lambert's law, where $E$ is the illuminance on the surface:

$$
L=\rho \frac{E}{\pi}
$$




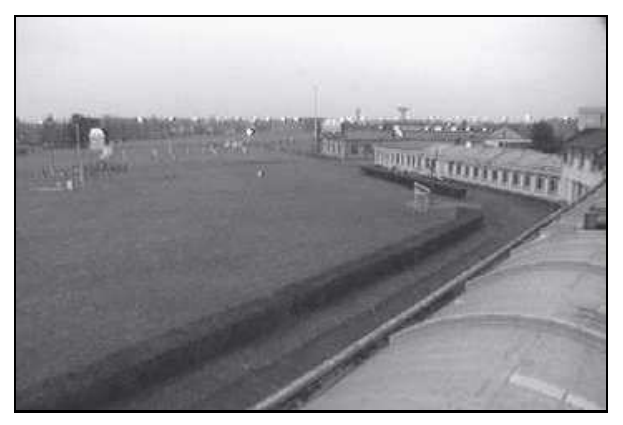

(a)

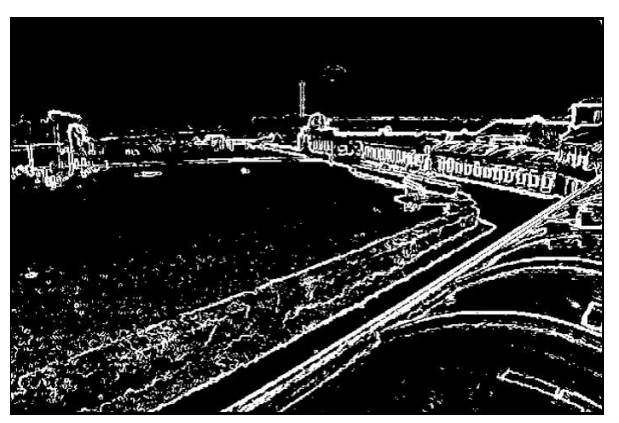

(b)

Figure 2: Module of Sobel gradient of the image: (a) image in good visibility conditions; (b) Module of Sobel gradient of the same image.

Hence, the expression of texture contrast for Lambertian objects is independent of illumination, and only depends on the object albedos $\rho_{1}$ and $\rho_{2}$, the distance $d$ and the extinction coefficient $k$ as shown in Equation 8:

$$
C_{\text {Texture,Lambert }}=\left(\rho_{2}-\rho_{1}\right) e^{-k d}
$$

The main advantage of using the texture contrast is that its value is robust to variations of illumination in the scene since it is expressed as a function of albedo, an intrinsic characteristic of materials. Therefore, according to Equation 8, this contrast is expected to be a very strong indicator of the meteorological visibility despite illumination changes. There is no need to assume that all objects in the scene are Lambertian, only to select those that are.

\subsection{Contrast as a Module of Sobel Gradient}

The contrast defined above is a one-dimensional concept. In our case, however, the image $\mathfrak{I}$ is twodimensional. The module of Sobel gradient, which indicates the value of the largest change from bright to dark at each pixel, is calculated with Equation 9:

$$
G=\sqrt{G_{x}^{2}+G_{y}^{2}}
$$

The horizontal and vertical gradients, respectively $G_{x}$ and $G_{y}$, are calculated by the convolution of the masks given in Equation 10:

$$
G_{x}=\left(\begin{array}{ccc}
+1 & 0 & -1 \\
+2 & 0 & -2 \\
+1 & 0 & -1
\end{array}\right) * \mathfrak{I} \quad \text { and } \quad G_{y}=\left(\begin{array}{ccc}
+1 & +2 & +1 \\
0 & 0 & 0 \\
-1 & -2 & -1
\end{array}\right) * \mathfrak{I}
$$

The outcome of this processing is illustrated in Figure 2. The original image is shown in Figure 2(a) and the gradient image, with edges enhanced as a direct result of the Sobel operator, is presented in Figure 2(b).

\subsection{Segmentation of Lambertian Surfaces}

Calculating the gradient of the image has been explained above. Those gradients which are most robust against illumination changes are extracted by selecting Lambertian surfaces within the scene. The best indicators of visibility variations are determined via this method, as shown with Equation 8. In practice, segmenting Lambertian areas in an image can be achieved by seeking the best linear correlation between 


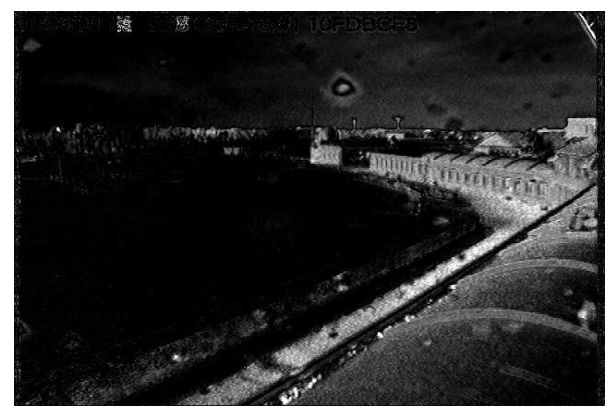

Figure 3: Confidence that the pixels belongs to Lambertian area. The intensity determines the level of correlation between pixel intensity and scene illumination over time.

the intensity changes of each pixel over time and the variations of illumination characterized by the sky luminance $L_{S k y}$. The probability $P_{i, j}^{L}$ that the surface at pixel $(i, j)$ is Lambertian can be calculated using the temporal correlation of Bravais-Pearson:

$$
P_{i, j}^{\mathrm{L}}=\operatorname{corr}\left(L_{i, j}, L_{\mathrm{Sky}}\right)
$$

This is illustrated in Figure 3 where the intensity denotes high correlation and, as a consequence, high probability for the surfaces to be Lambertian. More precisely, it is possible to demonstrate using clear-sky photometric models that such a method, inspired by (Koppal and Narasimhan, 2006; Andersen et al., 2006; Seon Joo, 2008), detects a set of pixels, which include pixels belonging to North-oriented Lambertian surfaces. This segmentation especially allows discarding the specular reflections, such as sunlight on smooth surfaces, as well as shadows created by the movement of the sun during the day.

\section{Estimation of Meteorological Visibility}

\subsection{Visibility Estimation based on Robust Gradient}

Let the estimator $E^{\text {all }}$ equal the sum of all existing gradients in the image, absent any consideration of reflection (for the time being). This allows using Equation 12 and also corresponds to precedent in the literature (Xie et al., 2008; Liaw et al., 2009). Now, let us consider the estimator of visibility $E^{\mathrm{L}}$ based on the sum of the module of Sobel gradient, weighted by the confidence $P^{\mathrm{L}}$ of the pixel to belong to a Lambertian area, defined by Equation 13.

$$
\begin{aligned}
E^{\text {all }} & =\sum_{(i, j) \in \mathfrak{I}} C_{0_{i, j}} e^{-k d_{i, j}} \\
E^{\mathrm{L}} & =\sum_{(i, j) \in \mathfrak{I}} P_{i, j}^{\mathrm{L}} C_{0_{i, j}} e^{-k d_{i, j}}
\end{aligned}
$$

where $(i, j)$ denotes the coordinates of an image pixel.

To adjust the response function of the visibility estimator given by Equation 13, an empirical and nonlinear model described by Equation 14 is given. This function is the response of the estimator $E^{\mathrm{L}}$ according to changes in visibility conditions $V_{\text {Met }}$ obtained by a visibility meter. The exponential relationship between the contrast and the meteorological visibility distance, expressed in Equations 6 and 13, explains the nonlinearity of the function. Therefore, the response of the estimator $\tilde{E}^{\mathrm{L}}$ of Equation 14 is adjusted by refining 
its parameters $A$ and $B$. This is done by minimizing the quadratic error between the response function and the cloud of points relating the visibility estimator $E^{\mathrm{L}}$ from the image and the measured meteorological optical range $V_{\text {Met }}$ :

$$
\tilde{E}^{L}=A+B \log \left(V_{M e t}\right)
$$

\subsection{Correlation as a Reliability Indicator of Visibility Estimation}

When the quadratic error is minimal, the correlation factor between estimator and visibility is close to 1 . So, estimating the visibility $V_{\text {Met }}$ by inversing the function $\tilde{E}^{\mathrm{L}}$ will be closer to the reference values given by the visibility meter. The correlation factor constitutes an indicator of reliability in estimating this response function $\tilde{E}^{\mathrm{L}}$.

\subsection{Error due to Model Fitting}

Parameters A and B must be adjusted so as to minimize the quadratic error $\chi^{2}$ between the measured visibility $V_{\text {Met }}$ and the visibility estimated by the function $\tilde{E}^{\mathrm{L}}\left(V_{\mathrm{Met}}, A, B\right)$ with Equation 15 :

$$
\chi^{2}=\frac{1}{N} \sum_{n=1}^{N}\left[V_{\operatorname{Met}_{n}}-\tilde{E}^{\mathrm{L}}\left(V_{\operatorname{Met}_{n}}, A, B\right)\right]^{2}
$$

\subsection{Weighted Fitting for Low Visibilities}

Most of the time, images of low visibility will be rare compared to images of good visibility. Because the proposed model is empirical, this drives the largest error of the estimation in the more sparse low visibility data set. Therefore the curve fitting is weighted by giving more confidence to cases of low visibility. Since the error increases linearly with visibility, the inverse of the accuracy $\sigma_{V_{\text {Met }}}$ is used as a confidence factor. This typically corresponds to $10 \%$ of the value of visibility $V_{\text {Met }}$. The results are shown in Figure 5.

$$
\chi^{2}=\frac{1}{N} \sum_{n=1}^{N} \frac{1}{\sigma_{V_{\mathrm{Met}_{n}}}}\left[V_{M t_{n}}-\tilde{E}^{\mathrm{L}}\left(V_{M t_{n}}, A, B\right)\right]^{2}
$$

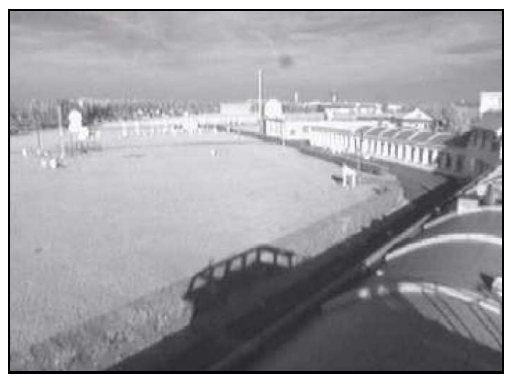

(a)

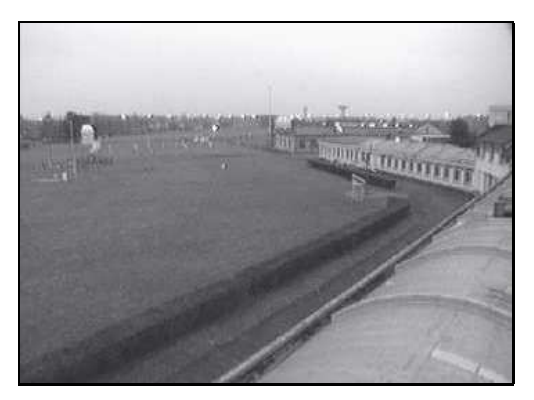

(b)

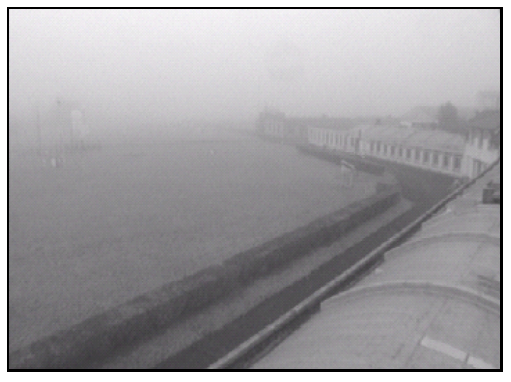

(c)

Figure 4: Examples of images taken over several months under lighting conditions from 0 to $10,000 \mathrm{~cd}^{\mathrm{m}} \mathrm{m}^{-2}$ and visibility conditions from 0 to $50 \mathrm{~km}$ : (a) Sunny day with shadows, (b) cloudy day, (c) low visibility. 


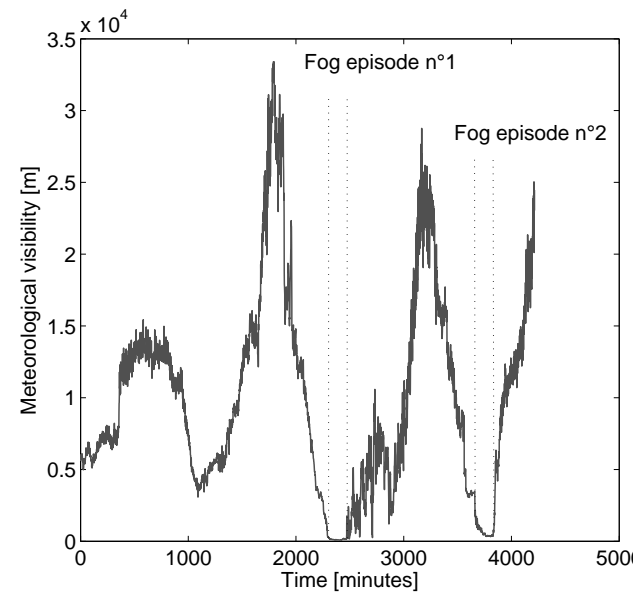

(a)

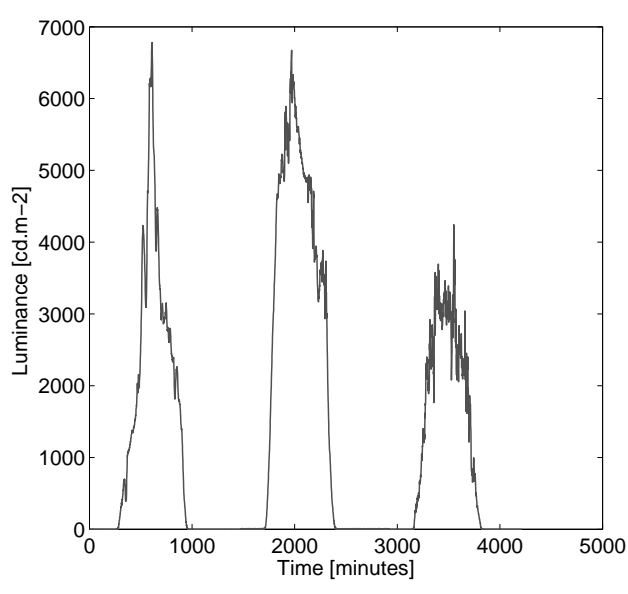

(b)

Figure 5: Data collection: (a) Meteorological optical range variation and (b) sky luminance variation during three days.

\section{Results}

\subsection{Image and Data Collection}

Visibility and lighting data have been collected over several months. These data were matched with images taken from a camera. Indeed, a meteorological observatory was instrumented with a CCTV camera and a digital video recorder. The camera has the same quality as a typical roadside camera (cf. section 2.2): 570TVL and a dynamic range of 8 bits per pixel. Images were acquired every 10 minutes for several months, with sky luminance between 0 and $10,000 \mathrm{~cd} \cdot \mathrm{m}^{-2}$ and meteorological optical range between 80 $\mathrm{m}$ and $50 \mathrm{~km}$. Sample images with different weather conditions are shown in Figure 4. The luminance data were collected by means of a luminance meter (Degreane Horizon LU320), and the visibility data were obtained by means of a scatterometer (Degreane Horizon DF320). Both instruments are common meteorological measurement systems often found on airports. Their operating principle was recalled in the background section. Sample data are shown in Figure 5. Among the collected data, we selected a subset, which correspond to two fog episodes. During these periods, the meteorological visibility distance dropped very low. The data used for experimental evaluation are thus made of 150 images (approximately 48 hours of observation spread on three different days).

\subsection{Qualitative Evaluation}

The sum of the module of Sobel gradient computed from the images is plotted as a function of the measured meteorological visibility in Figure 6(a). An instability and dispersion of the response of the estimator $E^{\text {all }}$ can be observed. This instability is related to the change in lighting conditions, and this directly affects the values of object luminance in the scene and therefore it affects the resulting gradients. The instability is also related to the different reflections of sunlight on glass or other smooth, non-Lambertian surfaces. Because the imaged scene contains these elements, the module of Sobel gradient of the entire image cannot be a robust indicator of the measured meteorological visibility.

Results for the estimator $\tilde{E}^{\mathrm{L}}$ are shown in Figure 6(b) with the continuous blue line. Points representing the visibility estimator $E^{\mathrm{L}}$ as a function of the measured visibility $V_{\text {Met }}$ follow an empirical law which 


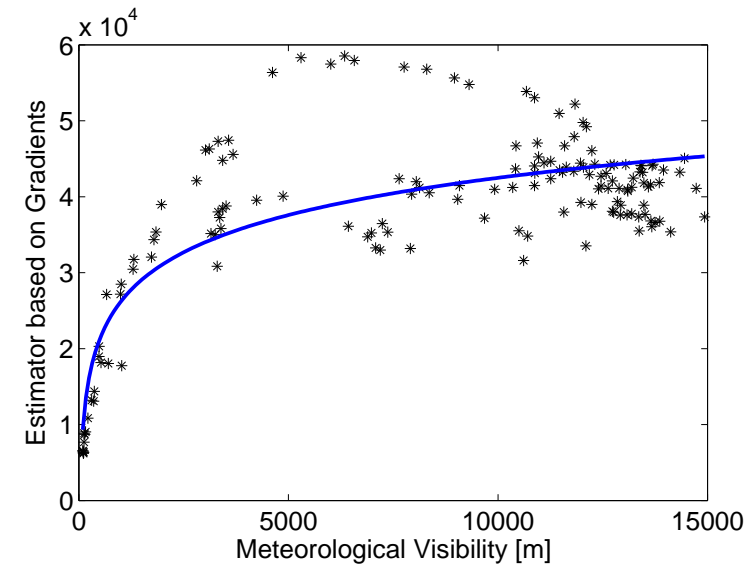

(a)

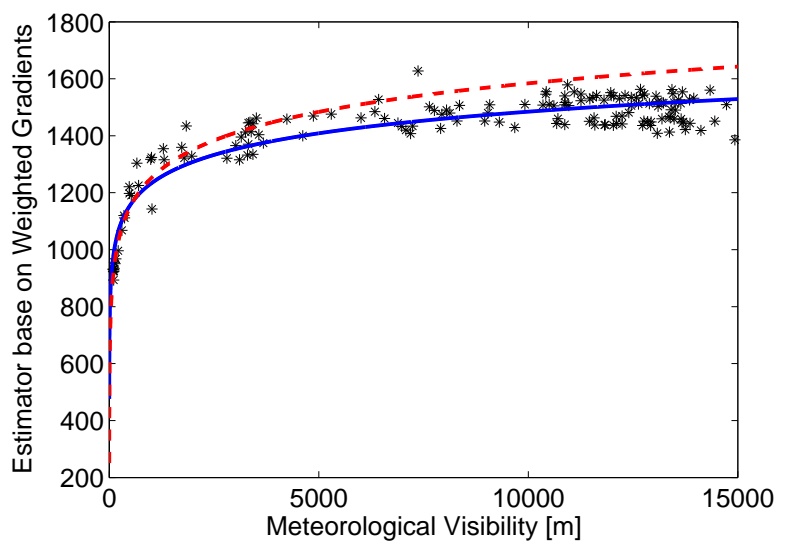

(b)

Figure 6: Logarithmic model fitting of the plot of the estimators as a function of reference visibilities: (a) $E^{\text {all }}$, sum of the module of Sobel gradient in the entire image; (b) $\tilde{E}^{\mathrm{L}}$, sum of the module of Sobel gradients weighted by the confidence of pixels to belong to Lambertian surfaces represented by the continuous blue line; $\tilde{E}^{\mathrm{L}}$, sum of the module of Sobel gradient weighted by the confidence of pixels to belong to Lambertian surfaces and with weighted fit for low visibility distances represented by the dashed red line.

appears to be logarithmic. For visibility distances below $1.5 \mathrm{~km}$, the curve fit is weighted $\left(\sigma_{V_{\text {Met }}}=10 \%\right)$ so as to reduce the influence of data with very high visibility. Results of this weighted curve fitting are shown in Figure 6(b) with the dashed red line.

\subsection{Quantitative Evaluation}

Meteorological visibility was estimated using an empirical response function. The values obtained are given in Table 1 for different applications, along with the average relative error $\frac{\Delta V}{V}$ from the reference values measured by a visibility meter. Processing the whole image results in a correlation factor of 0.82 . For large visibility distances, this corresponds to an average relative error of 100 to $200 \%$, meaning that the visibility estimation is irrelevant. Using gradients in Lambertian surfaces and a weighted fit for low visibility distance as described in this paper brings the average relative error down to $25 \%$, which makes the estimation of the visibility more robust and reproducible over time. For visibility distances beyond 5 $\mathrm{km}$, the average relative error becomes $33 \%$ using the unweighted fit, and it is as low as $10 \%$ for visibility distances below $400 \mathrm{~m}$ using the weighted fit. Finally, an assessment of the visibility estimator is carried out, wherein the model is calibrated with a first fog episode and its predictions are compared with the data of the second fog episode. In this way, we try to replicate a real-world application. The results are given in the last line of Table 1. Compared to the use of all data for calibration, the error increases approximately of $10 \%$, which also corresponds to the uncertainty of the visibility meter itself.

\section{Emerging Applications}

\subsection{Air Pollution Mitigation}

Air pollution has negative effects on human health. Living or even attending school near busy roads have been proven to increase respiratory symptoms. A lowering of maximum speed limits has been proven effective to reduce air pollution related to traffic in the direct vicinity of highway (Dijkema et al., 2008). In case of sudden ozone or nitrogen dioxide pollution, temporary speed limits reduction are often posted or 


\begin{tabular}{cccccc}
\hline Application & Highway fog & Fog & Haze & Air quality & Correlation \\
\hline Visibility range & $0-400 \mathrm{~m}$ & $0-1,000 \mathrm{~m}$ & $1,000-5,000 \mathrm{~m}$ & $5,000-15,000 \mathrm{~m}$ & $R^{2}$ \\
(Number of images) & 13 images & 19 images & 26 images & 105 images & 150 images \\
\hline$E^{\text {all }}$ & $22 \%$ & $39 \%$ & $205 \%$ & $125 \%$ & 0.82 \\
\hline$\tilde{E}^{\mathrm{L}}$ before weighted fit & $11 \%$ & $53 \%$ & $60 \%$ & $\mathbf{3 3 \%}$ & 0.95 \\
\hline$\tilde{E}^{\mathrm{L}}$ after weighted fit & $\mathbf{1 0 \%}$ & $25 \%$ & $\mathbf{2 6 \%}$ & $48 \%$ & 0.90 \\
\hline$\tilde{E}^{\mathrm{L}}$ weighted fit on fog episode 1 & $19 \%$ & $\mathbf{2 0 \%}$ & $37.5 \%$ & $65 \%$ & 0.89 \\
\hline
\end{tabular}

Table 1: Average relative error $\Delta V / V$ expressed in $\%$ as a function of range of application. Here the correlation factor is between 0.89 and 0.95 and corresponds to an error of $25 \%$ (short ranges and weighted fit) to $33 \%$ (long ranges and unweighted fit). For highway fog with less than $400 \mathrm{~m}$ visibility, the error is reduced to $10 \%$ (weighted fit).

alternate traffic rules are decided. These two transport policies have been shown to have positive effects on air quality (Schillinger et al., 2007). Air quality sensors are mainly installed in cities. By using the proposed camera-based monitoring method, road operators have at their disposal a flexible solution to assess the air quality and to potentially implement new strategies aiming at maintaining the air quality along their road networks.

\subsection{Winter Maintenance}

The monitoring of meteorological visibility has different applications for winter maintenance (Nagata et al., 2008). First, the knowledge of a low visibility area is important for the safety of winter maintenance operations. Second, a sudden drop of the visibility can be symptomatic of heavy snow falls. The relationship between liquid equivalent snowfall rate and visibility has also been investigated (Rasmussen, 1999), which means that a camera-based visibility meter is potentially a good snow sensor. Finally, meteorological models have been developed to forecast pavement temperatures as well as snow height (Bouilloud et al., 2009). Nebulosity and fog are phenomena which alter the prediction, since the radiative transfer between the pavement and the air is changed. The assimilation of visibility data in these prediction models may be useful to increase the accuracy of forecasts.

\subsection{Fog Nowcasting}

The forecasting of the weather within the next six hours is often referred to as nowcasting. In this time range, it is possible to forecast smaller features such as individual showers and thunderstorms with reasonable accuracy, as well as other features too small to be resolved by a computer model. Guidard and Tzanos (2007) show that combining satellite-based forecasting of low clouds with terrestrial measurements of humidity allows computing a probability of fog occurrence. A camera-based visibility meter could easily substitute the measurement of the humidity. Indeed, using camera-based visibility estimation and meteorological data, Yasuhiro et al. (2011) shows that visibility can be predicted up to 15 minutes with $1-\mathrm{km}$ mesh meteorological data. Such camera-based nowcasting methods may be good solutions to allow the re-routing of vehicles before they reach a low-visibility area in a timely manner.

\subsection{Pile-up Prevention and Mitigation}

\subsubsection{Best Practice}

McLawhorn (2004) has proposed a review of best practices in terms of mitigation of highway visibility problems, in particular fog related issues. In this paper, a description of existing installations in the USA dedicated to driver alert in case of low visibility on the highway is proposed. Apart from fog dispersal techniques, the best practices are related to the timely alert of the drivers who approach a foggy area. Then, 


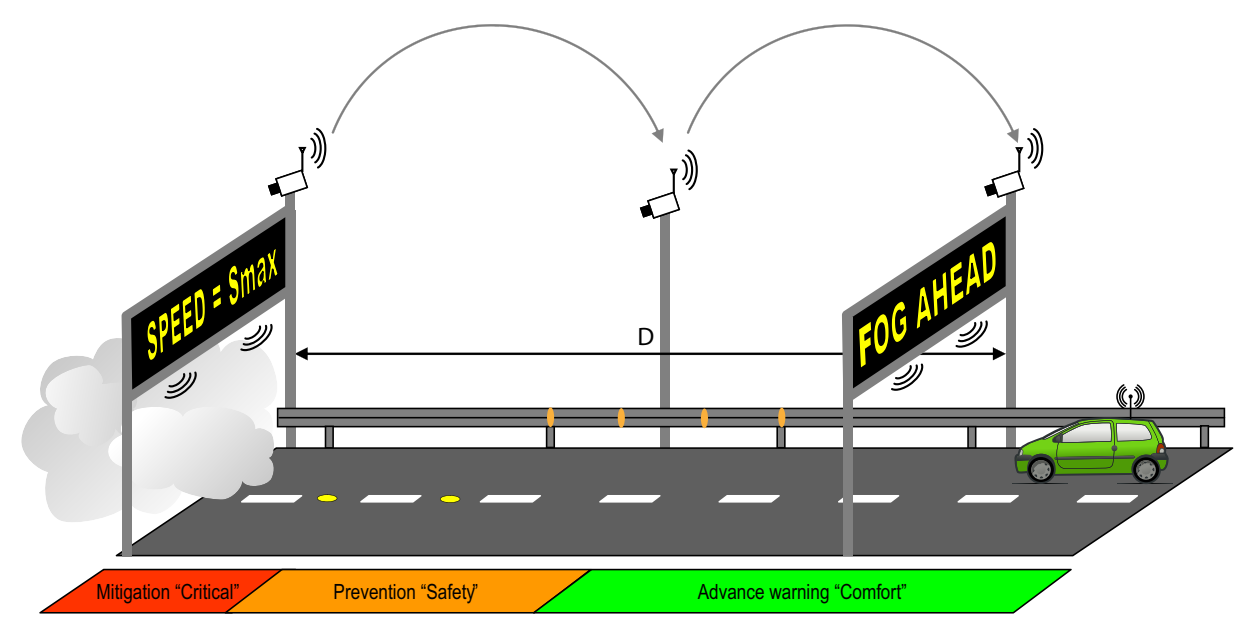

Figure 7: Futuristic decentralized fog-pilot, which makes use of CCTV cameras to monitor the visibility and allows optimizing the speed of drivers approaching a low visibility area, as well as the intensity of road studs.

depending on the fog density, different advisory speed limits may be posted. In the same time, the public lighting is adapted. The component of these systems are made of weather stations, CCTV cameras and Variable Message Signs (VMS). More recently, Caltrans has installed the "Fog Pilot" system in District 6, which provides a high-technology solution every $\frac{1}{4}$ mile along a 12 -mile $(20-\mathrm{km})$ portion of State Route 99. This centralized solution relies on the use of infrastructure-to-vehicle communications to alert drivers of sudden low speed areas.

\subsubsection{Decentralized Fog-Pilot}

In this section, we envision a decentralized fog-pilot, which makes use of CCTV cameras to monitor the visibility and allows optimizing the speed of drivers approaching a low visibility area, as well as the intensity of road studs. The different components of the fog-pilot are schematized in Figure 7. Based on best existing practices, its principle is to warn the drivers of a foggy area with enough time, so that their speed is adapted to the prevailing visibility distance when they reach the dangerous area.

Speed Management. The key aspect of this problem is to select the relevant Variable Message Sign (VMS), on which to display the information about an incoming foggy area. In this paragraph, we propose to compute the relative position of the VMS with respect to the position of the foggy area.

The stopping distance $D_{\text {stop }}$ for a vehicle is determined by its deceleration $\gamma$, which depends on the effective coefficient of friction between the tires and the road, and the driver's reaction time $T_{r}$ in a braking situation (Kiencke and Nielsen, 2000):

$$
D_{\text {stop }}=S_{0} T_{\mathrm{r}}+\frac{S_{0}^{2}}{2 \gamma}
$$

where $S_{0}$ denotes the initial speed of the vehicle.

By solving the equation $D_{\text {stop }}=V_{\text {Met }}$, we obtain the maximum speed $S_{\max }$ at which a driver should drive through fog, so as to be able to prevent a collision with a stopped vehicle:

$$
S_{\max }=-T_{r} \gamma+\sqrt{T_{\mathrm{r}}^{2} \gamma^{2}+2 \gamma V_{\mathrm{Met}}}
$$


The distance $D_{\text {brake }}$ covered by a vehicle with a deceleration $\gamma$ from initial speed $S_{0}$ to the target speed $S_{\max }$ is given by the following equation:

$$
D_{\text {brake }}=\frac{1}{2}\left[\frac{S_{\max }^{2}-S_{0}^{2}}{\gamma}\right]
$$

So as to obtain a smooth deceleration, e.g. $\gamma=3 \mathrm{~m} \cdot \mathrm{s}^{-2}$, the optimal distance $D_{\mathrm{vms}}$ between two VMS is:

$$
D_{\mathrm{vms}}=D_{\text {brake }}+S_{0} T_{\text {latency }}
$$

where $T_{\text {latency }}$ comprises the latency of the system to detect the low visibility area and to display the information on the relevant VMS. We thus have:

$$
T_{\text {latency }}=T_{\text {detection }}+T_{\text {display }}
$$

The time which is necessary to detect is usually very low compared to the time necessary to transmit and to display the information. In the case of our camera-based system, $T_{\text {detection }}$ is very small (lower than 0.1 second). In the case of a centralized system, the information must be validated by a human operator, which may take some additional time. In the case of a decentralized system, $T_{\text {display }}$ comprised the latency of the telecommunication system and might be of a few seconds. In the case of an incoming car driving at 130 $\mathrm{km} \cdot \mathrm{h}^{-1}$ approaching a section with $V_{\text {Met }}=50 \mathrm{~m}$, the distance $D_{\mathrm{vms}}$ is approximately equal to 400 meters for a smooth deceleration of $\gamma=3 \mathrm{~m} \cdot \mathrm{s}^{-2}$ and a very low latency (1 second). This optimal distance corresponds also to the definition of highway fog given in the introduction and validates the positioning of the VMS in the Fog-Pilot system.

Adaptive Road Lighting. In case of fog presence, the road lighting must also be adapted. First, streetlights are usually switched off. Adaptive lighting are put on the roadside and road studs on the pavement are switched on so as to better delineate the highway. In addition, we believe that intelligent road studs (Boys and Green, 1997) should be able to adapt their intensity with respect to the visibility distance.

The luminance of a road stud perceived by a driver at distance $d$ in case of fog is given by Allard's Law (CIE, 1987):

$$
L_{r} \propto \frac{I_{m}}{d^{2}} \exp (-3 k d) \approx \frac{I_{m}}{d^{2}} \exp \left(-\frac{3 d}{V_{\mathrm{Met}}}\right)
$$

where $I_{m}$ denote the maximum intensity of the luminous source (see Figure 8 ). To obtain the same luminance in fog as in clear conditions, it is enough to compensate for the decrease of light intensity caused by fog. By reversing Equation 22, we found that the intensity of a road stud must increased by a factor $\exp \left(\frac{3 d_{0}}{V_{\text {Met }}}\right)$. In this equation, $d_{0}$ denotes the distance at which the intensity of the road stud is perceived at the maximum ( 40 meters in case of a road stud dedicated to highway applications, i.e. $\varepsilon \approx 2^{\circ}$ and $h=1.2 \mathrm{~m}$ in Figure 8).

\section{Discussion}

\subsection{Qualitative Comparison with other Methods}

It is quite difficult to compare meteorological visibility estimation methods, because the data sets and the reference sensor differ from one method to another. Nevertheless, we have shown that taking Lambertian areas into account is more robust than relying on the whole image gradients. In this way, we improve the previous methods proposed by Xie et al. (2008) and Liaw et al. (2009). Compared to previous methods, we are able to estimate visibility ranges down to very short ranges like (Hautière et al., 2008), as well as up 


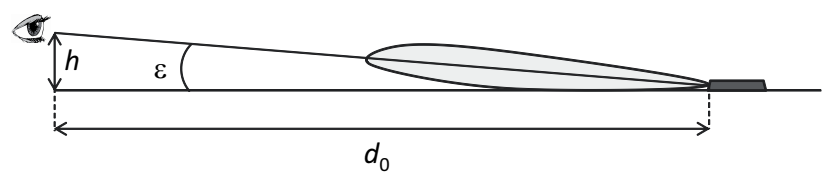

Figure 8: Geometry related to the luminous intensity distribution of a road stud at grazing angle $\varepsilon$. With this geometry, $d_{0}$ denotes the distance at which the intensity of the road stud is perceived at the maximum. It is equal to 40 meters for a car driver $(h=1.2$ $\mathrm{m})$ looking at a roadstud dedicated to highway applications $\left(\varepsilon \approx 2^{\circ}\right)$.

to long ranges like Liaw et al. (2010). We are (among) the first to propose a non-linear fitting to estimate the meteorological visibility distance. Unlike methods which detect known targets in the landscape, e.g. (Bäumer et al., 2008), we do not systematically underestimate the meteorological visibility distance. We do not rely on high resolution either since we use a camera which is already massively deployed on highway networks. Finally, we are not sensitive to the geometric calibration of the camera contrary to (Hautière et al., 2008), since we compute a global visibility descriptor instead of a local one.

\subsection{Perspectives}

Despite the good properties of the methods and the promising results, the proposed model still suffers two main limitations. First, fixed camera is a requirement for the here proposed method which is intended to operate with roadside cameras such as those used for traffic surveillance. Second, the method does not deal currently with rapid changes in the field of view caused by traffic. Third, the seasonality also impacts the amount of gradients in the image. The second and third limitations can be circumvented by using background modelling methods so as to constantly update the model, as previously proposed by Hautière et al. (2008).

To implement this method of visibility estimation on a specific site, calibrating the logarithmic response curve is mandatory. In this aim, the simplest method consists in matching image contrasts with visibility and luminance data collected by reference sensors (visibility meter and luminance meter) during at least one foggy episode. For a massive deployment of the method on many different sites, more dedicated work is needed to simplify the calibration process so as to get rid of the reference sensors. A first model-driven approach aiming at replacing the empirical logarithmic formula is proposed by Hautière et al. (2011). It is a promising solution. However, the experimental results show that our data-driven approach still gives better results in terms of relative error.

\section{Conclusion}

This study proposes a robust empirical law for estimating the meteorological visibility in daylight by means of a typical CCTV camera. The methodology presented in this paper is to link meteorological visibility to the sum of the module of Sobel gradient weighted by the confidence of the pixels to belong to Lambertian surfaces. It is demonstrated and validated that the proposed estimator is robust to changes in lighting conditions, and that any variation in measurement results are due to the variation of visibility in the atmosphere. Applying this estimator on real images acquired under a variety of visibility and lighting conditions, an estimated atmospheric visibility was obtained and then compared and validated with reference data collected with a meteorological instrument.

The approach for estimating visibility was also tested and validated under a large range of visibility and lighting conditions. It showed the relevance and the reproducibility of the approach. We believe therefore 
that this method for estimating meteorological visibility is easily deployable using the camera network already installed alongside highways throughout the world and therefore of high impact to traffic safety at marginal cost. Once deployed, this concept should increase the quality and the spatial accuracy of the visibility information and could feed weather forecasting systems. Importantly, our system may serve to inform drivers of relevant speed limits under low visibility conditions.

In future work, we will express errors in estimating visibility as a function of camera characteristics to ascertain the accuracy with which visibility can be estimated with current and future CCTV systems. We believe, however, that our work has given both a fundamental and practical basis to consider deployment of our potentially life-saving real-time roadside visibility meters.

\section{Acknowledgments}

The work presented in this paper is co-funded by Ifsttar and Météo-France. The authors wish to thank Jacques Parent du Chatelet for his contribution to the supervision of this work.

\section{References}

Abdel-Aty, M., Ekram, A.-A., Huang, H., Choi, K., 2011. A study on crashes related to visibility obstruction due to fog and smoke. Accident Analysis and Prevention 43, 1730-1737.

Andersen, M., Jensen, T., Madsen, C., 2006. Estimation of dynamic light changes in outdoor scenes without the use of calibration objects. In: International Conference on Pattern Recognition (ICPR'06). Vol. 4. pp. 91-94.

Bouilloud, L., Martin, E., Habets, F., Boone, A., Le Moigne, P., Livet, J., Marchetti, M., Foidart, A., Franchistéguy, L., Morel, S., Noilhan, J., Pettré, P., 2009. Road surface condition forecasting in france. Journal of Applied Meteorology and Climatology 48 (12), 2513-2527.

Boys, J., Green, A., 1997. Intelligent road-studs - lighting the paths of the future. IPENZ Transactions 24 (1), $33-41$.

Bäumer, D., Versick, S., Vogel, B., 2008. Determination of the visibility using a digital panorama camera. Atmospheric Environment 42, 2593-2602.

CIE, 1987. International Lighting Vocabulary. No. 17.4.

Committee on Haze in National Parks and Wilderness Areas, 1993. Protecting visibility in national parks and wilderness areas. National Research Council, Washington, DC.

Dijkema, A., van der Zee, S., Brunekreef, B., van Strien, R., 2008. Air quality effects of anurban highway speed limit reduction. Atmospheric Environment 42, 9098-9105.

Guidard, V., Tzanos, D., 2007. Analysis of fog probability from a combination of satellite and ground observation data. Pure and Applied Geophysics 164, 1207-1220.

Hagiwara, T., Ota, Y., Kaneda, Y., Nagata, Y., Araki, K., 2006. A method of processing CCTV digital images for poor visibility identification. Transportation Research Records: Journal of the Transportation Research Board 1973, 95-104.

Hallowell, R., Matthews, M., Pisano, P., 2007. An automated visibility detection algorithm utilizing camera imagery. In: 23rd Conference on Interactive Information and Processing Systems for Meteorology, Oceanography, and Hydrology (IIPS), San Antonio, TX, Amer. Meteor. Soc.

Hautière, N., Bigorgne, E., Bossu, J., Aubert, D., 2008. Meteorological conditions processing for vision-based traffic monitoring. In: International Workshop on Visual Surveillance, European Conference on Computer Vision.

Hautière, N., Babari, R., Dumont, E., Brémond, R., Paparoditis, N., March 2011. Lecture Notes in Computer Science, Computer Vision - ACCV 2010. Vol. 6495. Springer, Ch. Estimating Meteorological Visibility using Cameras: A Probabilistic ModelDriven Approach, pp. 243-254.

Hunfeld, F., Hauser, U., Kruse, K., April 2011. Die Höllenwand. Stern (16), 106-116.

Hyslop, N. P., 2009. Impaired visibility: the air pollution people see. Atmospheric Environment, 43 (1), 182-195.

Jacobs, N., W., B., Fridrich, N., Abrams, A., Miskell, K., Brswell, B., Richardson, A., Pless, R., 2009. The global network of outdoor webcams: Properties and apllications. In: ACM International Conference on Advances in Geographic Information Systems.

Kang, J., Ni, R., Andersen, G. J., 2008. Effects of reduced visibility from fog on car-following performance. Transportation Research Record: Journal of the Transportation Research Board (2069), 9-15.

Kiencke, U., Nielsen, L., 2000. Automotive Control Systems. Springer. 
Koppal, S., Narasimhan, S., 2006. Clustering appearance for scene analysis. In: IEEE Computer Society Conference on Computer Vision and Pattern Recognition (CVPR'06). pp. 1323 - 1330.

Liaw, J.-J., Lian, S.-B., Chen, R.-C., 2009. Atmospheric visibility monitoring using digital image analysis techniques. In: Jiang, X., Petkov, N. (Eds.), International Conference on Computer Analysis of Images and Patterns. Vol. 5702 of LNCS. pp. $1204-1211$.

Liaw, J.-J., Lian, S.-B., Huang, Y.-F., Chen, R.-C., 2010. Using sharpness image with haar function for urban atmospheric visibility measurement. Aerosol and Air Quality Research 10, 323-330.

Mac Carley, C. A., 2005. Methods and metrics for evaluation of an automated real-time driver warning system. Transportation Research Record: Journal of the Transportation Research Board (1937), 87-95.

McLawhorn, N., 2004. Mitigating highway visibility problems. In: National Highway Visibility Conference.

Middleton, W., 1952. Vision through the atmosphere. University of Toronto Press.

Nagata, Y., Hagiwara, T., Araki, K., Kaneda, Y., Sasaki, H., 2008. Application of road visibility information system to winter maintenance. Transportation Research Records: Journal of the Transportation Research Board 2055, 128-138.

Pauley, P., Baker, N., Barker, E., 1996. An observational study of the "interstate 5" dust storm case. Bull. Amer. Meteor. Soc. 77, 693-720.

Pejovic, T., Williams, V. A., Noland, R. B., Toumi, R., 2009. Factors affecting the frequency and severity of airport weather delays and the implications of climate change for future delays. Transportation Research Record: Journal of the Transportation Research Board (2139), 97-106.

Perry, A. H., Symons, L. J., 1991. Highway Meteorology. University of Wales Swansea, Swansea, Wales, United Kingdom.

Rasmussen, Roy M., J. V. J. C. B. M. C. M., 1999. The estimation of snowfall rate using visibility. Journal of Applied Meteorology and Climatology 38 (10), 1542-1563.

Roquelaure, S., Tardif, R., Remy, S., Bergot, T., 2009. Skill of a ceiling and visibility local ensemble prediction system (leps) according to fog-type prediction at paris-charles de gaulle. Airport. Weather and Forecasting 24, 1511-1523.

Schillinger, C., Deprost, R., Riviere, E., 2007. Assessment of potential emergency measures in Alsace to reduce air pollution peaks. Recherche Transports Sécurité 27 (2), 120-127.

Seon Joo, K., 2008. Radiometric calibration with illumination change for outdoor scene analysis. In: IEEE Conference on Computer Vision and Pattern Recognition.

Shepard, F., 1996. Reduced Visibility Due to Fog on the Highway. No. 228.

Thach, T. Q., Wonga, C. M., , C. K.-P., Chaua, Y., Chunga, Y. N., Oub, C. Q., Yanga, L., Hedleya, A. J., 2010. Daily visibility and mortality: Assessment of health benefits from improved visibility in Hong-Kong. Environmental Research 110 (6), 617-623.

Whiffen, B., Delannoy, P., Siok, S., May 2004. Fog: Impact on road transportation and mitigation options. In: National Highway Visibility Conference, Madison, Wisconsin, USA.

Xie, L., Chiu, A., Newsam, S., 2008. Estimating atmospheric visibility using general-purpose cameras. In: Bebis, G. (Ed.), International Symposium on Visual Computing, Part II. Vol. 5359 of LNCS. pp. 356-367.

Yasuhiro, N., Hagiwara, T., Takitani, K., Kawamura, F., Kaneda, Y., Sakai, M., 2011. Development of a visibility forecast model based on a road visibility information system (RVIS) and meteorological data. In: Transportation Research Board Annual Meeting, Washington DC, USA. No. Paper 11-2342. 